\title{
«Lücken in Prävention \\ und Früherkennung schliessen»
}

\section{Interview: Bruno Kesseli}

Dr. med. et lic. phil., Chefredaktor

\author{
Am 8. November 2016 wurde die «Nationale Strategie Herz- und Gefässkrankheiten, \\ Hirnschlag und Diabetes» (HGHD-Strategie) in Bern lanciert. Im folgenden Inter- \\ view ziehen Felix Mahler und Augusto Gallino, vormaliger bzw. aktueller Präsident \\ von CardioVasc Suisse ${ }^{1}$, eine Zwischenbilanz.
}

\section{Wie war die Resonanz auf die Lancierung der HGHD-Strategie?}

Felix Mahler: Die Strategie wurde sehr wohlwollend aufgenommen. Die Gastreferenten Regierungsrat Thomas Heiniger, Präsident der GDK², und Pascal Strupler, Direktor des $\mathrm{BAG}^{3}$, fanden lobende Worte zu unserer Initiative. Die Kantone wissen, dass die Prävention in der Schweiz gefördert werden sollte, und sehen sich durch unser Anliegen darin bestärkt. Das BAG hat eine enge Kooperation mit seiner umfassenden NCD-Strategie $^{4}$ angeboten.

\section{Worin liegt die besondere Bedeutung dieser natio- nalen Strategie?}

Augusto Gallino: Die HGHD-Strategie stellt wahrscheinlich ein Unikum dar, indem durch einen partizipativen Prozess von Vertretern aus mehreren Fachgebieten (Kardiologie, Angiologie, Neurologie, Diabetes) unter Mitwirkung weiterer ärztlicher und nichtärztlicher Fachgebiete ein gemeinsames Konzept erarbeitet wurde.

CardioVasc Suisse ist eine Allianz von 26 national

tätigen kardiovaskulären medizinischen

Fachgesellschaften sowi Förder- und Patientenorganisationen. Sie treten für Initiativen, Programme und Massnahmen zur wirksamen Bekämpfung von Herz-Kreislauf-Erkrankungen und Diabetes ein.

2 Schweizerische Konferenz der kantonalen Gesundheitsdirektorinnen und -direktoren.

3 Bundesamt für Gesundheit.

4 «Nationale Strategie zur Prävention nichtübertragbarer Krankheiten".

\section{Warum braucht es in der medizinisch bestens} versorgten Schweiz eine solche nationale Strategie? Mahler: Der Schwerpunkt der Strategie liegt nicht auf der Versorgung, obwohl die Verkürzung der Wege zur Akuttherapie von Herzinfarkt und Hirnschlag auch ein Anliegen ist. Es gilt vielmehr Lücken in Prävention und Früherkennung der Krankheitsrisiken und in der Datenlage zu schliessen.

\section{Welche Hauptziele verfolgt die Strategie?}

Gallino: Die HGHD-Strategie versteht sich als komplementär zur NCD-Strategie, indem sie sich nicht nur mit primär präventiven Massnahmen befasst, sondern auch mit sekundärer und tertiärer Prävention sowie

\section{Die Gesprächspartner}

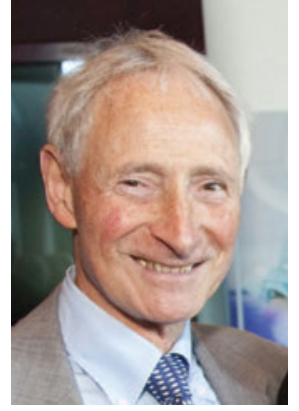

Felix Mahler

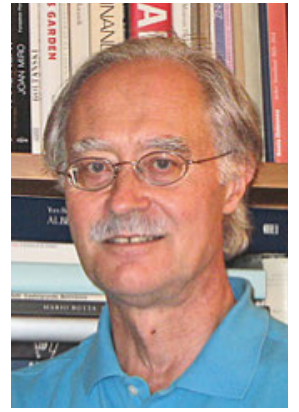

Augusto Gallino
Geboren 1940. Spezialarzt FMH für Angiologie und Innere Medizin Chefarzt und Ordinarius für Angiologie am Inselspital Bern bis zur Emeritierung 2005. Kaderarzt für Angiologie am Spital Männedorf bis Ende 2015. Lehrbuch über angiologische Interventionen. Ex-Präsident Schweizerische Gesellschaft für Angiologie, Gründungsmitglied der Union Schweizerischer Gesellschaften für Gefässkrankheiten. Stiftungsrat Schweizerische Herzstiftung. Präsident CardioVasc Suisse von 2004 bis 2016

Geboren 1950. Chefarzt Innere Medizin, Angiologie und Kardiologie am Spital Bellinzona von 1992 bis 2015 Titularprofessor an der Universität Zürich seit 1998. Ex-Präsident der Schweizerischen Gesellschaft für Angiologie. Direktionsmitglied der Schweizerischen Herzstiftung bis Ende 2016 und Präsident von deren Forschungskommission. Vorstandsmitglied verschiedener nationaler (AGLA) und internationaler Gesellschaften (European Society of Cardiology, International Society of Cardiovascular Pharmacology). Leiter der Arbeitsgruppe Gefässe bei der HGHDStrategie. Hauptinteressen in klinischer Forschung und kardiovaskulärer Strategie. Präsident CardioVasc Suisse seit Anfang 2017. 
Optimierung beziehungsweise Koordination der Betreuung von manifest und insbesondere chronisch erkrankten Patienten.

«Die HGHD-Strategie stellt wahrscheinlich ein Unikum dar, indem durch einen partizipativen Prozess [...] ein gemeinsames Konzept erarbeitet wurde.»

\section{Wer hat den Anstoss zur Erarbeitung der Strategie gegeben?}

Mahler: Es handelte sich um eine Initiative von CardioVasc Suisse, einer Dachorganisation von 26 kreislauforientierten Fachgesellschaften, Stiftungen und $\mathrm{Pa}$ tientenorganisationen mit besonderer Unterstützung durch die Schweizerische Herzstiftung.

\section{Wie lief der Erarbeitungsprozess ab? Wie lange dauerte er?}

Gallino: Der Ansatz war bewusst partizipativ. Es gab vier krankheitsbezogene Arbeitsgruppen und einen Workshop zur Erarbeitung der allen gemeinsamen Ziele. Der Prozess dauerte deshalb zweieinhalb Jahre. Eindrücklich war es, in dieser Zeit den festen Willen aller Beteiligten zu spüren, zu gemeinsamen Zielen und Lösungen zu kommen.

\section{Auf welchem Weg und mit welchen Mitteln sollen die Ziele erreicht werden?}

Gallino: Durch eine zentrale, multidisziplinäre Steuerungskommission der Strategie sollen in partizipativem Stil den sogenannten Playern der Strategie Anstösse zur Entwicklung und Durchführung neuer Projekte gegeben werden. Die Player können Fachgesellschaften, Patientenorganisationen oder Behörden sein. Natürlich sollen auch die schon bestehenden Aktivitäten unterstützt werden.

\section{Dies klingt etwas abstrakt. Könnten Sie dazu ein konkretes Beispiel geben?}

Gallino: Ein Beispiel ist das Vorhofflimmern, das in zunehmendem Mass zu spät erkannt wird. Da es nicht nur den Bereich Herz und Gefässe betrifft, sondern durch Bildung von Embolien auch Hirnschläge verursacht, wird dessen Früherkennung und Behandlung

«Eindrücklich war es, in dieser Zeit den festen Willen aller Beteiligten zu spüren, zu gemeinsamen Zielen und Lösungen zu kommen.»

mit konsequenter Antikoagulation als allgemeine, das heisst gebietsübergreifende Massnahme, eine hohe Priorität erhalten.
Mahler: Es existieren bereits verschiedene Register zur Erfassung von Interventionen im kardiologischen, angiologischen und gefässchirurgischen Bereich. Eine Koordination dieser Register durch die Fachbereiche Herz und Gefässe soll geprüft werden.

\section{Wo sehen Sie die grössten Schwierigkeiten bei der Umsetzung der Strategie?}

Mahler: Bei den beschränkten Ressourcen der beteiligten Fachgesellschaften und Patientenorganisationen. Zudem wird über Prävention zwar viel berichtet, dennoch werden noch sehr wenige finanzielle Ressourcen auf allen Ebenen dafür zur Verfügung gestellt. Das Sprichwort «Vorbeugen ist besser als heilen» muss sich erst noch durchsetzen.

\section{Als wie gross stufen Sie die Gefahr ein, dass diese Strategie ein Papiertiger bleibt?}

Gallino: Diese Gefahr ist real, wenn der jetzige Impetus der bisherigen Player nicht genutzt wird. Bereits am 1. Februar dieses Jahres wurde die erste Sitzung der Steuerungskommission von CardioVasc Suisse abgehalten. Somit ist der erste Schritt in der Umsetzung der Strategie bereits erfolgt.

\section{«Das Sprichwort «Vorbeugen ist besser} als heilen` muss sich erst noch durchsetzen.»

Die Schweizerische Hypertonie-Gesellschaft (SHG) hat in einer ersten Reaktion bemängelt, dass der Bluthochdruck, aber auch Dyslipidämien und der Tabakkonsum in der Strategie nicht den angezeigten Stellenwert erhalten hätten. Ist dieser Vorwurf berechtigt?

Gallino: Die Bedeutung der spezifischen Risikofaktoren, insbesondere der Hypertonie, ist uns voll bewusst. Sie sollen aber nicht nur einzeln, sondern integral erfasst und behandelt werden, beispielsweise durch Screeningaktionen für Hypertonie, Dyslipidämie und Diabetes. Wir nehmen aber den call for action der SHG sehr ernst und werden auf sie zugehen.

Inwiefern und auf welche Weise werden die Ärztinnen und Ärzte eingebunden?

Gallino: Der Beitrag der Ärzteschaft läuft vor allem über die Kontakte mit den verschiedenen Fachgesellschaften. Die Grundversorger spielen eine entscheidende Rolle. Sie sind ja das Rückgrat der medizinischen Betreuung in der Schweiz. Eine spezifische, aber auch breit angelegte Strategie wie die HGHD muss in ein holistisches Konzept integriert werden. 
Können Sie an einem Beispiel verdeutlichen, in welcher Weise die Grundversorger eingebunden werden könnten?

Mahler: Die Grundversorger spielen beim Erfolg solcher wichtigen Projekte durch ihre Präsenz an der Front des schweizerischen Gesundheitssystems eine entscheidende Rolle. Daher hoffen wir sehr auf ihre Teilnahme in der Steuerungskommission: Gerade bei Projekten wie zum Beispiel dem Screening auf Vorhofflimmern ist ihre Unterstützung und Mitarbeit von grosser Bedeutung.

\section{«Die Grundversorger spielen eine} entscheidende Rolle.

Sie sind ja das Rückgrat der medizinischen

Betreuung in der Schweiz."

\section{Die Ziele der Strategie sollen über interprofessionelle Zusammenarbeit erreicht werden. Wie sieht diese aus?}

Mahler: Diese soll gefördert werden, etwa durch Schulungen und durch vermehrte Delegationsmöglichkeiten medizinischer Aufgaben an nichtärztliche Berufsgruppen.

Gibt es spezielle Massnahmen, um die Interprofessionalität zu fördern?

Gallino: Nehmen Sie zum Beispiel die Behandlung von chronischen Ulzera: Es ist suboptimal, ein solches chronisches Leiden ohne eine multidisziplinäre, interprofessionelle Equipe zu behandeln.

\section{An welchen Kriterien wird der Erfolg der Strategie gemessen?}

Gallino: Als wir diese «Übung» gestartet haben, waren wir schockiert, wie wenige statistische Daten über die kardiovaskuläre Betreuung vorhanden waren. Eines der wichtigsten Ziele der HGHD-Strategie ist diesem Problem gewidmet.

\section{Wie wird nun an der Umsetzung der Strategie weitergearbeitet?}

Mahler: CardioVasc Suisse wird sich unter dem neuen Präsidium von Augusto Gallino vornehmlich der Umsetzung der Strategie widmen. Es sollen prioritäre Projekte der an der Strategie beteiligten Player identifiziert werden. Ausserdem wird die Zusammenarbeit

"CardioVasc Suisse wird sich unter dem neuen Präsidium von Augusto Gallino vornehmlich der Umsetzung der Strategie widmen.»

mit der NCD-Strategie gesucht. Nach Abklärung von Finanzierungsmöglichkeiten von Seiten öffentlicher und/oder privater Partner sollten erste Aktionen schon 2017 gestartet werden können. Wir sind der Schweizerischen Herzstiftung sehr dankbar für ihre Bereitschaft, die HGHD-Strategie weiter zu unterstützen. 